\title{
31 Years of Clinical Hemorheology and Microcirculation
}

The founder and first Editor-in-Chief of our journal, Alfred L. Copley, started Clinical Hemorheology in 1980 with Pergamon Press. The first issue contained a very important overview by Alfred L. Copley "On the endothelial fibrin lining, fibrinogen clotting extra vivum and in vivo, surface hemorheology and pathological conditions". With number 6 of Volume 11, Alfred L. Copley completed his activities as Executive Editor of Clinical Hemorheology. The Executive Editorial Office moved to Karlsruhe, Germany, with Siegfried Witte and Jean-Francois Stoltz as Editors-in-Chief.

Starting with Vol. 13, No. 1 a new subtitle was chosen: "An International Journal of Blood Flow, Vessel Wall, Interstitium: Transport and Interaction". This came with an emphasis on the importance of both, science and applications of clinical hemorheology, comprising not only the blood and its components but also all those tissues in permanent or temporary contact with blood/blood components including interstitial tissues.

In 1996 the title of the journal was broadened to "Clinical Hemorheology and Microcirculation" in accordance with the trend of the clinical research in our field, being focussed on the terminal blood vessels as the anatomically and functionally most important areas of hemorheology.

Until 2001 Siegfried Witte together with Jean-Francois Stoltz put a great effort into making Clinical Hemorheology and Microcirculation a publication platform for scientists working in blood rheology worldwide.

From 2000 on, Sandro Forconi and Friedrich Jung worked as managing editors and they took over the duty as Editors-in-Chief in 2003 together with Jean-Francois Stoltz and Hideyuki Niimi.

Persistent efforts of the editors and the continuously advancing contributions of the participating scientists, considerably improved by the very fruitful scientific exchange during the periodically held international meetings of the European Society of Clinical Hemorheology and Microcirculation, of the International Society of Clinical Hemorheology and of the International Society for Biorheology resulted in a tremendous increase in the Impact Factor of our journal starting from 0.29 in the year 2000 up to 3.389 in 2012.

For the issue of Clinical Hemorheology and Microcirculation now released, opinion leaders in the different branches of our field were asked to review and give an overview of the most important and recent developments. We would like to thank all the authors for their excellent contributions and to thank the publisher IOS Press, especially, who supported all of our efforts to the best. 
F. Jung

Centre for Biomaterial Development and

Berlin-Brandenburg Centre for Regenerative Therapies

(BCRT), Helmholtz-Zentrum Geesthacht, Teltow, Germany

H. Niimi

National Cardiovascular Center Research Institute

Department of Biomedical Engineering Fujishiro-dai 5-7-1

Suita Osaka

Japan
S. Forconi

Siena-Toronto Centre

University of Siena

Banchi di Sotto 81

Siena, Italy

\section{J.F. Stoltz}

UMR CNRS 7563 Bioingénierie

Faculté de Médecine

Brabois BP 184

F-54500 Vandoeuvre lès Nancy

France 